\title{
Geminate and Nongeminate Recombination of Triplet Excitons Formed by Singlet Fission
}

\author{
Sam L. Bayliss, ${ }^{1}$ Alexei D. Chepelianskii, ${ }^{1,2}$ Alessandro Sepe, ${ }^{1}$ Brian J. Walker, ${ }^{1}$ Bruno Ehrler, ${ }^{1}$ \\ Matthew J. Bruzek, ${ }^{3}$ John E. Anthony, ${ }^{3}$ and Neil C. Greenham ${ }^{1, *}$ \\ ${ }^{1}$ Cavendish Laboratory, University of Cambridge, J. J. Thomson Avenue, Cambridge, CB3 OHE, United Kingdom \\ ${ }^{2}$ LPS, Université Paris-Sud, CNRS, UMR 8502, F-91405, Orsay, France \\ ${ }^{3}$ Department of Chemistry, University of Kentucky, Lexington, Kentucky 40506, USA
}

(Received 13 March 2014; revised manuscript received 2 May 2014; published 9 June 2014)

\begin{abstract}
We report the simultaneous observation of geminate and nongeminate triplet-triplet annihilation in a solution-processable small molecule TIPS-tetracene undergoing singlet exciton fission. Using optically detected magnetic resonance, we identify recombination of triplet pairs directly following singlet fission, as well as recombination of triplet excitons undergoing bimolecular triplet-triplet annihilation. We show that the two processes give rise to distinct magnetic resonance spectra, and estimate the interaction between geminate triplet excitons to be $60 \mathrm{neV}$.
\end{abstract}

DOI: 10.1103/PhysRevLett.112.238701

PACS numbers: 88.40.jr, 71.35.-y, 76.70.Hb

Singlet exciton fission [1,2] is a promising route to enhance both organic and inorganic solar cells [3-5], giving the potential to achieve power-conversion efficiencies well above the conventional Shockley-Queisser limit of around $33 \%$ [6,7]. Through singlet fission, a spin-zero singlet exciton created by absorption of a photon is transformed into two spin-one triplet excitons, which can be harvested in a solar cell to generate two electron-hole pairs from a single photon, which allows more efficient harnessing of highenergy photons from the solar spectrum. Since the fission process is spin conserving, it can be extremely fast and efficient [8-10], generating high yields of triplet pairs. These pairs are initially entangled due to formation from a spinless singlet exciton, and serve as a unique signature of the fission process $[11,12]$. To minimize energy losses in this carrier multiplication process, a singlet fission material would ideally have degenerate singlet and triplet-pair states. However, with this energy level configuration, the inverse process of singlet fission, triplet-triplet annihilation, becomes a possible recombination pathway, reducing the overall triplet yield. In solar cells, efficiently generating photocurrent from triplets formed through singlet fission remains problematic. It is therefore of great importance to study the processes of triplet recombination that compete with photocurrent generation [13], both at early and later times following fission, as well as to expand the class of materials known to undergo this process efficiently.

A material satisfying the energetic requirements for efficient singlet fission is tetracene, in which the singlet and triplet-pair states are nearly degenerate, permitting photoluminescence to be used as the experimental observable [14-19]. However, since tetracene is difficult to process from solution, it has limited practical application. TIPStetracene [bis(triisopropylsilylethynyl)tetracene, Fig. 1(c)] is a solution-processable tetracene derivative [20], and a candidate for singlet fission. However, it has different molecular packing compared to tetracene [21], which is an important factor in determining singlet fission rates $[22,23]$ and triplet-triplet interactions [24]. Understanding the generation and recombination of triplet excitons in new systems such as this is therefore an important aspect of advancing the field of singlet fission photovoltaics.

Here we exploit optically detected magnetic resonance to probe the presence and properties of triplet excitons in spincoated TIPS-tetracene films. By measuring the changes in photoluminescence under spin resonance, we identify the unique spin signatures of the fission process. We find evidence for an interaction within the triplet pair formed immediately after fission, and simultaneously observe the annihilation of triplets directly after fission, as well as from bimolecular encounters.

Optically detected magnetic resonance (ODMR) $[25,26]$ is a powerful technique to unambiguously identify triplet excitons since the spin dipole-dipole interaction between the electron and hole gives rise to characteristic broad magnetic resonance spectra $[27,28]$. This interaction allows triplets to be distinguished from other species in ODMR; this contrasts with all-optical techniques where the presence of triplet states can only be inferred indirectly. The interaction is governed by the zero-field splitting Hamiltonian $\hat{H}_{\text {ZFS }}=$ $D\left(\hat{S}_{Z}^{2}-\hat{\mathbf{S}}^{2} / 3\right)+E\left(\hat{S}_{X}^{2}-\hat{S}_{Y}^{2}\right)$, where $\hat{\mathbf{S}}=\left(\hat{S}_{X}, \hat{S}_{Y}, \hat{S}_{Z}\right)$ is the triplet spin operator, and $D$ and $E$ reflect the magnitude of this dipole-dipole interaction between the electron and hole. Frankevich et al. have demonstrated ODMR as an elegant tool to detect the spin-correlated, geminate, triplet pairs formed through the fission process in crystalline tetracene [29], and a theoretical treatment has been advanced by others, highlighting the role of molecular packing [16-18]. ODMR has also been applied to the more common scenario of nongeminate triplet-triplet annihilation in which 


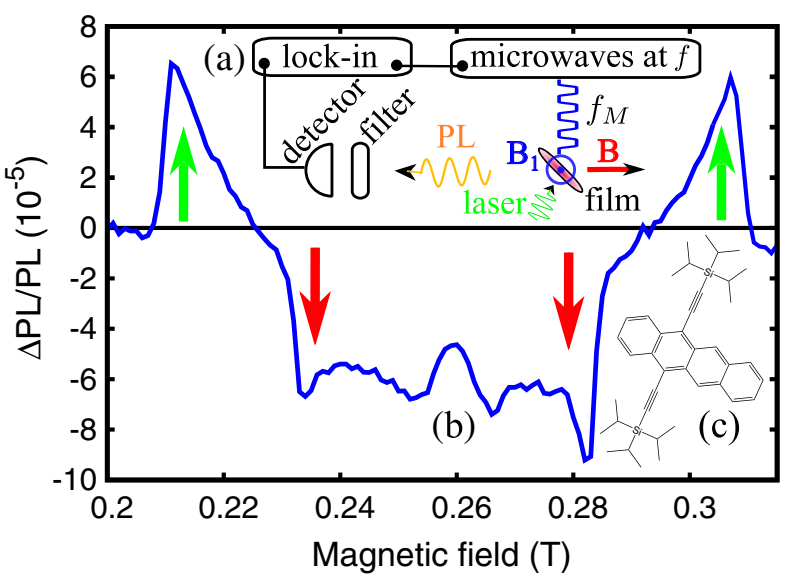

FIG. 1 (color online). (a) Experimental ODMR setup. (b) In-phase ODMR of spin-coated TIPS-tetracene films at $f=$ $7.28 \mathrm{GHz}$ and $f_{M}=17 \mathrm{~Hz}$. Both negative and positive ODMR features are visible, indicated by the inner (red) and outer (green) arrows, respectively. A small nonresonant offset has been subtracted. (c) Chemical structure of TIPS-tetracene.

two triplets recombine after a bimolecular encounter [30-33]. Since microwave pumping drives triplet-pairs out of the singlet configuration in the geminate case, but increases the number of singlets from recombination of nongeminate triplets, these two processes lead to resonant changes in photoluminescence with opposite sign, and so ODMR provides a sensitive way to distinguish them.

The experimental setup is outlined in Fig. 1(a). Films were prepared by spin coating from a chloroform solution onto glass substrates, and contained a mixture of crystalline and amorphous regions [21]. The films were placed on a microwave stripline operated between frequencies $f=6-18 \mathrm{GHz}$, providing the ac magnetic field $B_{1}$, and mounted inside an optically accessible cryostat magnet, providing the static magnetic field $B$, at an operating temperature of $1.4 \mathrm{~K}$. Excitation was provided by a $532 \mathrm{~nm}$ laser with intensity $I=110 \mathrm{~mW} / \mathrm{cm}^{2}$, and the integrated photoluminescence (PL), which extends from $550-700 \mathrm{~nm}$, was collected by a silicon photodetector, after removing the laser line with a $550 \mathrm{~nm}$ long-pass filter. Microwaves were square-wave modulated at frequency $f_{M}$, and the change in photoluminescence due to microwave transitions $(\Delta \mathrm{PL})$ was recorded as the static magnetic field was swept through resonance by monitoring the photodetector response at the microwave chopping frequency, using lock-in detection.

Figure 1(b) shows the microwave-induced changes in photoluminescence for the TIPS-tetracene films, normalized by the steady-state photoluminescence, for a low microwave chopping frequency $\left(f_{M}=17 \mathrm{~Hz}\right)$, where both positive and negative features are seen. The characteristic broad resonances identify that the features arise from triplet excitons, and provide initial evidence for singlet fission in this material. The presence of two separate features is also

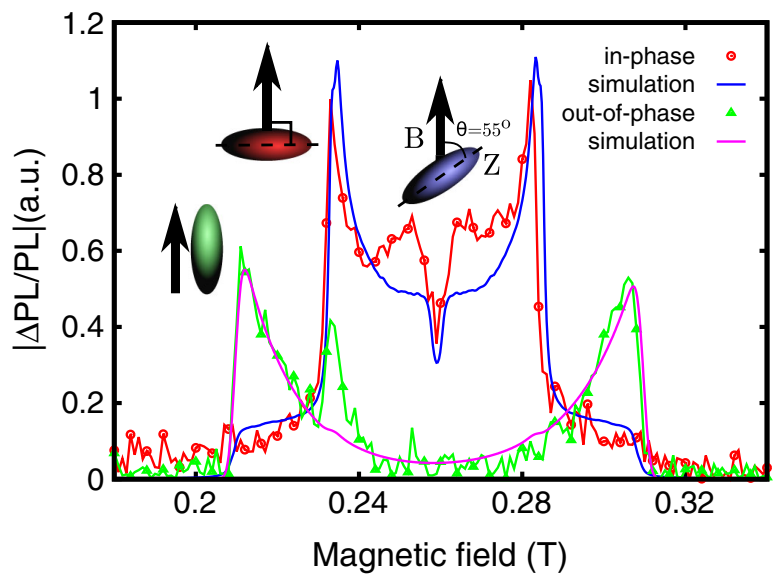

FIG. 2 (color online). ODMR at $317 \mathrm{~Hz}$ microwave chopping frequency where the two distinct line shapes are seen on the in-phase and out-of-phase lock-in channels, along with simulations for geminate and nongeminate triplet-triplet annihilation. (Experimental data taken at $f=7.28 \mathrm{GHz}$ and microwave power is $10 \mathrm{~dB}$ less than in Fig. 1). Details of the simulations can be found in the main text. The insets show the orientations of the triplet zero-field splitting tensor corresponding to the prominent features of the line shapes.

clear at a higher microwave chopping frequency of $317 \mathrm{~Hz}$ (experimental traces of Fig. 2), where the disparate patterns appear on separate lock-in channels. Since the negative inner features occur in phase, while the positive outer features occur out of phase, this indicates that the inner ODMR line shape corresponds to much faster kinetics than the outer line shape [34]. This was also seen on sweeping the microwave chopping frequency up to $50 \mathrm{kHz}$ at the magnetic fields corresponding to the two different features, where we found a relatively slow roll-off with frequency for the inner line shape, while the outer line shape exhibited a much more rapid roll-off (see Supplemental Material [21]). Using the chopping frequency $f_{M}^{*}$ at which the magnitude of the ODMR dropped to one half of its maximum value, the characteristic time scale $\tau=\sqrt{3} /\left(2 \pi f_{M}^{*}\right)$ was $25 \mu$ s for the inner peaks, and $690 \mu \mathrm{s}$ for the outer peaks [35]. Distinct recombination kinetics are also shown in the laser intensity dependences of the two features (Fig. 3): the inner line shape follows an approximately linear dependence of $\Delta \mathrm{PL}$ on laser intensity, while the outer line shape follows an approximately quadratic dependence. (The steady-state PL remained linear over this power range.) Based on the observed kinetics, we can assign the negative inner features (in-phase trace, Fig. 2) to a short-lived species undergoing a monomolecular recombination process, and the positive outer features (out-of-phase trace, Fig. 2) to a longer-lived species undergoing bimolecular recombination. Since we see no spin-one-half feature [36], we do not consider interactions with charges, such as triplet-polaron interactions $[37,38]$, to be significant, and therefore assign the ODMR to triplet-triplet recombination. 


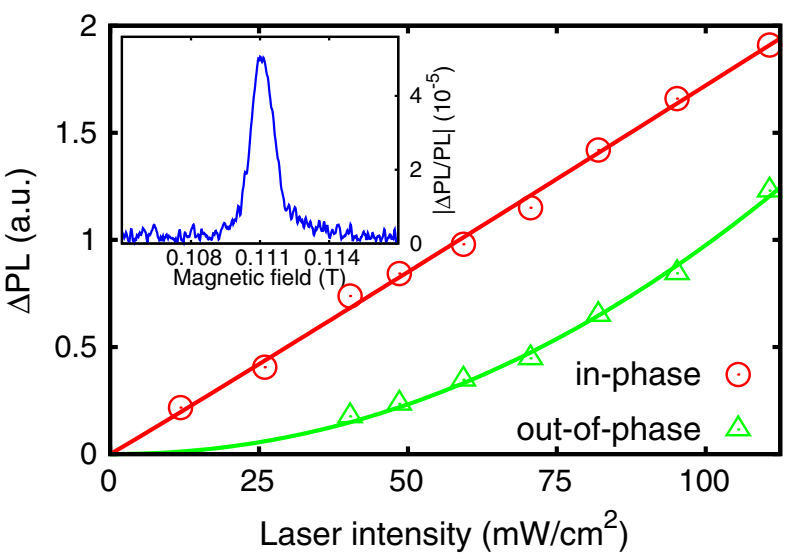

FIG. 3 (color online). Laser intensity dependence of the magnitude of the in-phase, and out-of-phase ODMR signals indicated in Fig. 2, along with fits of the form $\Delta \mathrm{PL} \propto I^{\alpha}$, where $I$ is the laser intensity, giving approximately linear $(\alpha=1.02 \pm$ $0.03)$ and quadratic $(\alpha=2.1 \pm 0.1)$ dependences for the in- and out-of-phase signals. (Data taken at $f=6.4 \mathrm{GHz}, f_{M}=317 \mathrm{~Hz}$, and the same microwave power as in Fig. 1.) Inset: magnitude of the half-field resonance under the same experimental conditions showing a single resonant field.

In a material where singlet fission is operative, two distinct triplet-triplet recombination pathways arise. Geminate triplet pairs may recombine to the emissive singlet state immediately following fission, or triplets may diffuse through the film and undergo bimolecular triplet-triplet annihilation. For a pair of identical, weakly interacting triplets, the nine triplet-pair energy eigenstates are not pure spin states, and at Zeeman energies much greater than their zero-field splitting parameters (and away from level degeneracies) consist of two singlet-quintet $(S Q)$ states with mixed singlet and quintet $(S=2)$ character, four pure quintet $(Q)$ states and three pure triplet $(T)$ states [39]. The latter form an isolated manifold due to symmetry considerations, and so we can consider only the states with singlet or quintet character [39]. Since singlet fission occurs from a singlet exciton, geminate triplet pairs will be formed in states with singlet character. If spin-lattice relaxation is slow compared to the pair lifetime, microwave transitions will act to transfer geminate pairs out of the singletcontaining states, decreasing the total population of triplet pairs that can recombine to the emissive singlet and resulting in a decrease in photoluminescence [Fig. 4(a)]. In addition, since a geminate triplet pair is formed from a single photon, this process should exhibit a monomolecular intensity dependence, as observed in Fig. 3. We therefore attribute the negative inner ODMR feature to a reduction in the population of short-lived, singlet-character geminate pairs. These pairs show a monomolecular decay and fast dynamics that is capped by the pair lifetime, consistent with the chopping frequency response.

Further evidence for this interpretation comes from the central dip in the inner ODMR feature, which deviates from (a) Geminate

(b) Non-geminate

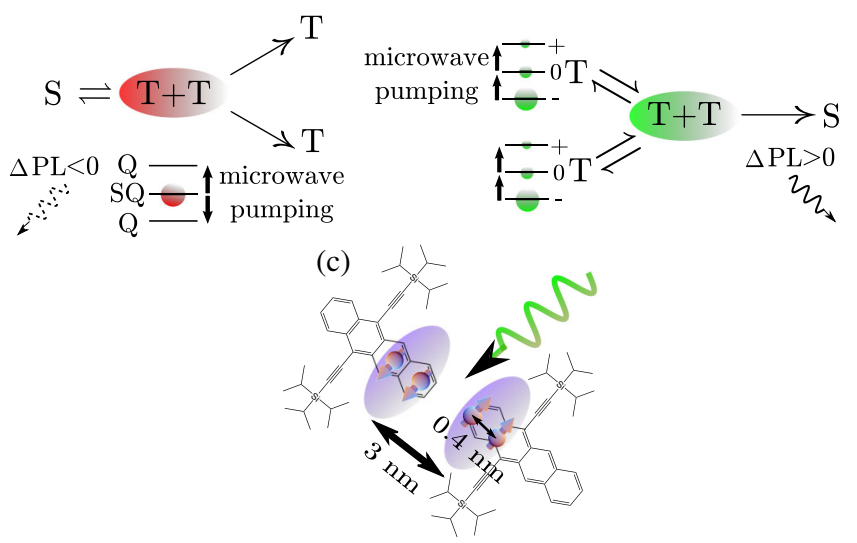

FIG. 4 (color online). Schematic of the geminate and nongeminate triplet-triplet annihilation processes. (a) In the geminate case, triplet pairs will be initially in one of the singlet-character $(S Q)$ states. Microwave pumping will transfer population to the nonemissive quintet $(Q)$ states, giving a negative ODMR, with monomolecular kinetics. (b) In the nongeminate case, microwave pumping will increase the population of emissive triplet pairs, giving a positive ODMR. (c) Summary of the triplet interaction lengths described in the main text.

a conventional triplet powder pattern [27]. As proposed by Frankevich [40], this behavior can result from the set of triplets that are oriented such that the singlet-quintet states become degenerate [which for a triplet with zero-field splitting parameter $E \approx 0$ will occur when the zero-field splitting tensor makes an angle of $\cos ^{-1}(1 / \sqrt{3}) \approx 55^{\circ}$ with the magnetic field, illustrated in Fig. 2]. At this degeneracy point, the zero-field splitting term vanishes, and even a weak triplet-triplet interaction will act to mix the singletquintet states to form pure singlet $(S=0)$ and quintet $(S=2)$ states, the transition between which is forbidden by the selection rule $\Delta S=0$. There can therefore be no transfer from or to the singlet manifold, and so the ODMR should approach zero at the center of the pattern (which corresponds to this degeneracy point).

We reproduced the geminate ODMR response, including the central dip, using a stochastic Liouville equation simulation for the triplet-pair density matrix $\hat{\rho}$ :

$$
\frac{\partial \hat{\rho}}{\partial t}=-\frac{i}{\hbar}\left[\hat{H}_{0}+\hat{H}_{1}(t), \hat{\rho}\right]+\hat{G}-\gamma \hat{\rho}-\gamma_{S}\left\{\hat{P}_{S}, \hat{\rho}\right\} .
$$

Here $\hat{H}_{0}$ is the static pair Hamiltonian consisting of the zero-field splitting $\left(\hat{H}_{\mathrm{ZFS}}\right)$ and Zeeman terms for the two triplets, and we introduce an intertriplet interaction term $J \hat{\mathbf{S}}_{1} \cdot \hat{\mathbf{S}}_{2}$ as an approximation to the interaction between the triplets $[12,41]$, where $J$ is the interaction strength and $\hat{\mathbf{S}}_{1}$, $\hat{\mathbf{S}}_{2}$ are the spin operators for the two triplets. $\hat{H}_{1}(t)$ is the ac Hamiltonian describing the microwave field, and $\hat{G}$ is the source term representing generation from the singlet. $\gamma$ is a spin-independent relaxation rate, and the final term 
represents the spin-dependent annihilation back to the emissive singlet state via the singlet projection operator $\hat{P}_{S}=|S\rangle\langle S|$, where $|S\rangle$ is the singlet state. (Quintet states as a final annihilation product are assumed to lie too high in energy $[42,43])$. The square and curly brackets denote the commutator and anticommutator, respectively. The ODMR is proportional to the microwave-induced change in singlet projection of the pair:

$$
\mathrm{ODMR} \propto\left[\operatorname{Tr}\left(\hat{P}_{S} \hat{\rho}^{(\text {on })}\right)-\operatorname{Tr}\left(\hat{P}_{S} \hat{\rho}^{\text {(off) })}\right],\right.
$$

where $\hat{\rho}^{\text {(on) }}$ and $\hat{\rho}^{\text {(off) }}$ are the pair density matrices with microwaves on and off, respectively. The resulting simulation is shown in Fig. 2. A good fit to the line shape is obtained with triplet zero-field splitting parameters $D=5.8 \mu \mathrm{eV}$ (corresponding to $D / g \mu_{B}=50 \mathrm{mT}$, where $g$ is the electron $g$ factor, and $\mu_{B}$ is the Bohr magneton) and $E \lesssim D / 40$. Since these parameters measure the dipole-dipole interaction between electron and hole, they give an estimate for the characteristic size of the exciton [27]. We find $r=3.8 \AA$, which corresponds to an electron-hole separation of around 1.5 benzene rings, and is comparable to the triplet size estimated theoretically for pentacene [44]. The central dip can only be reproduced by introducing a small intertriplet interaction $(J \approx D / 100)$, providing evidence for a weak, but finite, interaction of around $60 \mathrm{neV}$ within the triplet pair. Taking this interaction to be of dipole-dipole origin, which we expect to dominate over direct exchange interactions due to the localized nature of the triplet states, we can make a tentative estimate of the interaction length between the triplets as $r_{\mathrm{TT}}=r(4 D / J)^{1 / 3}$, where the factor of 4 arises since the interaction is between two spin-one particles, rather than two spin-one-half particles. This gives $r_{\mathrm{TT}} \approx 2.8 \mathrm{~nm}$, which is comparable to the TIPS-tetracene unit cell parameters of $\approx 1.5 \mathrm{~nm}$, determined by single crystal $\mathrm{x}$-ray diffraction, and consistent with a relatively localized triplet pair. These results therefore identify singlet fission in spin-coated TIPS-tetracene films, and indicate that it occurs with negligible thermal activation, since our experiments were performed at cryogenic temperatures.

The question then arises as to why the ODMR spectra occur with different line shapes. If the only difference between geminate and nongeminate triplet-triplet annihilation were the distribution of populations amongst spin sublevels, then we would expect overlapping resonances. In this case we would be unable to resolve the positive and negative features separately. However, we see distinct features, with the maxima of the outer "wings" appearing at double the separation of the inner maxima of the geminate pair, and so the two recombination processes must be distinguishable in another way.

Different zero-field splitting parameters arising from distinct triplets or from averaging over different sets of rotationally inequivalent molecules can be ruled out, since this would give rise to separated half-field resonances.
For example, a doubling of $D$ would lead to half-field resonances separated by around $10 \mathrm{mT}$, which we do not observe (see inset of Fig. 3), leading us to conclude that the two line shapes must arise from triplets with the same zero-field splitting parameters. This implies that the outer "wings" must therefore occur for triplets with their zero-field splitting tensors preferentially oriented along the magnetic field, which we simulate in Fig. 2 by including a preferential orientation of the triplets along the magnetic field in a triplet electron spin resonance powder pattern simulation [45]. The experimental line shape is reproduced using the orientational distribution function $P(\theta)=\exp \left[\lambda\left(3 \cos ^{2} \theta-1\right) / 2\right]$, where $\theta$ is the angle between the zero-field splitting tensor $\hat{Z}$ axis and the magnetic field, using an order parameter of $\lambda=4$.

The possibility that this anisotropy arises from preferential molecular orientation within the film [46] can be ruled out, since this would show a characteristic change of the line shapes as the film is rotated in the magnetic field, which we do not observe. The observed anisotropy is consistent, however, with anisotropic spin relaxation of thermalized triplets, due to a thermal modulation of the spin dipole-dipole interaction $[47,48]$. This leads to much longer spin-lattice relaxation times for triplets with their zero-field splitting tensor oriented along the direction of the magnetic field, than for those oriented perpendicular to the field $[47,48]$. We therefore assign the positive outer ODMR features to bimolecular encounters of thermalized triplets following separation after singlet fission [Fig. 4(b)]. At low temperatures, thermalized triplets will preferentially occupy the lowest Zeeman state $|-\rangle$, and the bimolecular encounter of these "cold" triplets has a low probability to reform a singlet, due to spin selection rules [49]. Microwave pumping of these free triplets will act to equalize the populations of the spin sublevels, increasing the effective spin temperature. Encounters involving triplet pairs with a total spin projection of zero therefore become more probable for these "hot" triplets, resulting in an increase in the annihilation rate to the emissive singlet, and hence an increase in photoluminescence. Since triplets oriented along the magnetic field experience a much slower spin-lattice relaxation rate, they can be effectively pumped, and so dominate the ODMR spectrum. Triplets oriented away from the magnetic field experience a much faster spin-lattice relaxation rate, and therefore give a much weaker contribution to the ODMR spectrum, resulting in the observed line shape. This interpretation is consistent with the different kinetics between geminate and nongeminate recombination, which allows the anisotropic relaxation rates to be revealed in the slower bimolecular process, but not in the fast geminate annihilation pathway.

In summary, using optically detected magnetic resonance we have identified singlet fission in a solutionprocessable small molecule, and have simultaneously observed the signatures of geminate and nongeminate 
triplet-triplet annihilation. Within the geminate triplet pair, we find evidence for a finite interaction of approximately $60 \mathrm{neV}$, and we estimate the intertriplet separation to be around $3 \mathrm{~nm}$. Our results highlight that when singlet and triplet-pair states are approximately degenerate, triplettriplet annihilation can be an important recombination pathway for fission-generated triplets.

This work was supported by the Engineering and Physical Sciences Research Council [Grants No. EP/ J017361/1 and No. EP/G060738/1]. A. D. C. acknowledges support from the E. Oppenheimer Foundation and St. Catharine's College, Cambridge. We thank Tim Skinner for experimental assistance. J. E. A. and M. J. B. thank the NSF (CMMI-1255494) for support of their efforts. Part of the work was conducted at beam line D1 at the Cornell High Energy Synchrotron Source (CHESS), supported by the NSF and NIH/NIGMS via NSF Grant No. DMR133220. We thank D.-M. Smilgies for help.

*ncg11@ cam.ac.uk

[1] M. B. Smith and J. Michl, Chem. Rev. 110, 6891 (2010).

[2] M. B. Smith and J. Michl, Annu. Rev. Phys. Chem. 64, 361 (2013).

[3] D. N. Congreve, J. Lee, N. J. Thompson, E. Hontz, S. R. Yost, P. D. Reusswig, M. E. Bahlke, S. Reineke, T. Van Voorhis, and M. A. Baldo, Science 340, 334 (2013).

[4] B. Ehrler, M. W. B. Wilson, A. Rao, R. H. Friend, and N. C. Greenham, Nano Lett. 12, 1053 (2012).

[5] P. J. Jadhav, P. R. Brown, N. Thompson, B. Wunsch, A. Mohanty, S. R. Yost, E. Hontz, T. Van Voorhis, M. G. Bawendi, V. Bulović et al., Adv. Mater. 24, 6169 (2012).

[6] W. Shockley and H. J. Queisser, J. Appl. Phys. 32, 510 (1961).

[7] M. C. Hanna and A. J. Nozik, J. Appl. Phys. 100, 074510 (2006).

[8] J. Johnson, A. Nozik, and J. Michl, J. Am. Chem. Soc. 132, 16302 (2010).

[9] M. W. B. Wilson, A. Rao, J. Clark, R. S. S. Kumar, D. Brida, G. Cerullo, and R. H. Friend, J. Am. Chem. Soc. 133, 11830 (2011).

[10] S. T. Roberts, R. E. McAnally, J. N. Mastron, D. H. Webber, M. T. Whited, R. L. Brutchey, M. E. Thompson, and S. E. Bradforth, J. Am. Chem. Soc. 134, 6388 (2012).

[11] W.-L. Chan, J. R. Tritsch, and X.-Y. Zhu, J. Am. Chem. Soc. 134, 18295 (2012).

[12] J. J. Burdett, G. B. Piland, and C. J. Bardeen, Chem. Phys. Lett. 585, 1 (2013).

[13] N. J. Thompson, E. Hontz, D. N. Congreve, M. E. Bahlke, S. Reineke, T. Van Voorhis, and M. A. Baldo, Adv. Mater. 26, 1366 (2014).

[14] R. Groff, P. Avakian, and R. Merrifield, Phys. Rev. B 1, 815 (1970).

[15] J. J. Burdett and C. J. Bardeen, J. Am. Chem. Soc. 134, 8597 (2012).

[16] T. Barhoumi, J. L. Monge, M. Mejatty, and H. Bouchriha, Eur. Phys. J. B 59, 167 (2007).

[17] T. Barhoumi, F. Henia, S. Romdhane, J. L. Monge, M. Mejatty, and H. Bouchriha, Eur. Phys. J. B 38, 395 (2004).
[18] M. Mejatty, J. Monge, V. Ern, and H. Bouchriha, Phys. Rev. B 43, 2558 (1991).

[19] R. E. Merrifield, P. Akavian, and R. Groff, Chem. Phys. Lett. 3, 155 (1969).

[20] S. A. Odom, S. R. Parkin, and J. E. Anthony, Org. Lett. 5, 4245 (2003).

[21] See Supplemental Material at http://link.aps.org/ supplemental/10.1103/PhysRevLett.112.238701 for details.

[22] R. J. Dillon, G. B. Piland, and C. J. Bardeen, J. Am. Chem. Soc. 135, 17278 (2013).

[23] G. B. Piland, J. J. Burdett, D. Kurunthu, and C. J. Bardeen, J. Phys. Chem. C 117, 1224 (2013).

[24] H. Bouchriha, V. Ern, J. Fave, C. Guthmann, and M. Schott, Chem. Phys. Lett. 53, 288 (1978).

[25] J. Shinar, Laser Photonics Rev. 6, 767 (2012).

[26] Triplet State ODMR Spectroscopy: Techniques and Applications to Biophysical Systems, edited by R. H. Clarke (Wiley, New Jersey, 1982).

[27] J. A. Weil and J. R. Bolton, Electron Paramagnetic Resonance (Wiley, New Jersey, 2007), Chap. 6, pp. 162-187.

[28] L. S. Swanson, J. Shinar, and K. Yoshino, Phys. Rev. Lett. 65, 1140 (1990).

[29] E. Frankevich and S. Kubarev, Triplet State ODMR Spectroscopy: Techniques and Applications to Biophysical Systems (Wiley, New Jersey, 1982), Chap. 5, pp. 137-183.

[30] A. Benfredj, F. Henia, L. Hachani, S. Romdhane, and H. Bouchriha, Phys. Rev. B 71, 075205 (2005).

[31] L. Hachani, A. Benfredj, S. Romdhane, M. Mejatty, J. L. Monge, and H. Bouchriha, Phys. Rev. B 77, 035212 (2008).

[32] D. Stich, F. Späth, H. Kraus, A. Sperlich, V. Dyakonov, and T. Hertel, Nat. Photonics 8, 139 (2013).

[33] M. Cai, Y. Chen, J. Shinar, O. Mitrofanov, C. Kloc, and A. P. Ramirez, Proc. SPIE Int. Soc. Opt. Eng. 7415, 74151Y (2009).

[34] V. Dyakonov, G. Rösler, M. Schwoerer, and E. L. Frankevich, Phys. Rev. B 56, 3852 (1997).

[35] G. Dellepiane, C. Cuniberti, D. Comoretto, G. F. Musso, G. Figari, A. Piaggi, and A. Borghesi, Phys. Rev. B 48, 7850 (1993).

[36] T. D. Nguyen, G. Hukic-Markosian, F. Wang, L. Wojcik, X.-G. Li, E. Ehrenfreund, and Z. V. Vardeny, Nat. Mater. 9, 345 (2010).

[37] M.-K. Lee, M. Segal, Z. G. Soos, J. Shinar, and M. A. Baldo, Phys. Rev. Lett. 94, 137403 (2005).

[38] W. J. Baker, D. R. McCamey, K. J. van Schooten, J. M. Lupton, and C. Boehme, Phys. Rev. B 84, 165205 (2011).

[39] R. E. Merrifield, Pure Appl. Chem. 27, 481 (1971).

[40] E. Frankevich, A. Pristupa, and V. Lesin, Chem. Phys. Lett. 47, 304 (1977).

[41] H. Benk and H. Sixl, Mol. Phys. 42, 779 (1981).

[42] P. Sibani and J. B. Pedersen, Phys. Rev. B 26, 2584 (1982).

[43] A. Suna, Phys. Rev. B 1, 1716 (1970).

[44] S. Sharifzadeh and P. Darancet, J. Phys. Chem. Lett. 4, 2197 (2013).

[45] S. Stoll and A. Schweiger, J. Magn. Reson. 178, 42 (2006).

[46] S. Cambre, J. D. Ceuster, E. Goovaerts, A. Bouwen, and H. Detert, Appl. Magn. Reson. 31, 343 (2007).

[47] J. Wolfe, Chem. Phys. Lett. 10, 212 (1971).

[48] P. Fischer and A. Denison, Mol. Phys. 17, 297 (1969).

[49] J. Wang, A. Chepelianskii, F. Gao, and N. C. Greenham, Nat. Commun. 3, 1191 (2012). 